\title{
Gezielte Therapie: alte und neue Hoffnungsträger
}

\author{
Andreas Hochhaus $^{\mathrm{a}} \quad$ Diana Lüftner $^{\mathrm{b}} \quad$ Andreas Mackensen $^{\mathrm{c}}$ \\ a Abteilung Hämatologie/Onkologie, Klinik für Innere Medizin II, Universitätsklinikum Jena, \\ ${ }^{\mathrm{b}}$ Klinik für Hämatologie, Onkologie und Tumorimmunologie, Charité Campus Benjamin Franklin, Berlin, \\ ${ }^{c}$ Medizinische Klinik 5, Hämatologie und Internistische Onkologie, Universitätsklinikum Erlangen, Deutschland
}

\section{Die Entwicklung gezielt wirkender Medikamente am Beispiel von Imatinib}

\section{Verantwortlicher Autor: Andreas Hochhaus, Jena}

Der Tyrosinkinasehemmer Imatinib (Glivec ${ }^{\circledR}$, Novartis Pharma GmbH, Nürnberg, Deutschland) wurde 2001 zunächst für die Behandlung von Patienten mit chronischer myeloischer Leukämie (CML) zugelassen, erfuhr danach mehrfache Zulassungserweiterungen und ist zu einer Standardmedikation in den Indikationen CML und gastrointestinaler Stromatumor (GIST) geworden. Der Wirkstoff wird nach Auslaufen des Patentschutzes in Form generischer Präparate zur Verfügung stehen.

Imatinib ist ein Paradebeispiel für die erfolgreiche Entwicklung zielgerichteter Therapien («Targeted Therapies») mit sogenannten «Small Molecules». Mit seiner Zulassung gelang ein Durchbruch in der Therapie der CML. Diese Form der Leukämie, die erstmals 1845 fast zeitgleich von R. Virchow und dem schottischen Arzt H. Bennett beschrieben worden ist, hatte bis Ende der 1960er-Jahre eine sehr ungünstige Prognose mit einem medianen Überleben von etwa 3 Jahren. Die Beschreibung des Philadelphia-Chromosoms als zytogenetisches Korrelat der CML [1] war die wichtigste Voraussetzung für die Entwicklung zielgerichteter Therapien. Ein Folgeprodukt der Mutation im Philadelphia-Chromosom $(\mathrm{Ph}+)$ ist das Fusionsprotein Bcr-Abl, das eine gesteigerte Tyrosinkinaseaktivität aufweist und den Organismus zur verstärkten Proliferation hämatopoetischer Vorläuferzellen anregt. Daraus ergab sich der - zunächst hypothetische - Therapieansatz, durch Hemmung der gesteigerten Enzymaktivität die Krankheitsprogredienz zu verringern. Die Grundlagenforschung lieferte mit der Entdeckung des murinen abl-Leukämievirus [2] und der Beschreibung von c- $a b l$ als Proto-Onkogen [3] weitere Bausteine für die
Medikamentenentwicklung. An der Etablierung von Mausmodellen zur Induktion der CML durch Expression von BcrAbl waren auch europäische Wissenschaftler beteiligt $[4,5]$. Als Therapieoptionen aus der Imatinib-Ära standen hauptsächlich Hydroxyurea und Interferon (IFN)- $\alpha$ zur Verfügung. Mit IFN- $\alpha$ wurden bei etwa $10-15 \%$ der Patienten komplette zytogenetische Remissionen erreicht, die mit einem verlängerten medianen Gesamtüberleben von $72 \%$ nach 10 Jahren verbunden waren [6].

Die Entwicklung vom Imatinib begann in den 1980er-Jahren, zunächst in den Forschungslaboratorien von Ciba-Geigy. In präklinischen Untersuchungen kristallisierte sich Imatinib - seinerzeit noch STI571 genannt - als potenter und selektiver Hemmer der Bcr-Abl-Tyrosinkinaseaktivität heraus. Imatinib war zwar nicht die erste Substanz aus der Gruppe der zielgerichteten Therapien gegen Tyrosinkinasen (der Antikörper Trastuzumab war schon verfügbar), wohl aber die erste orale Therapie mit diesem Wirkansatz. Entsprechend groß waren die Erwartungen an die neue Substanz.

\section{Beschleunigtes Zulassungsverfahren}

Bereits die ersten klinischen Phase-I/II-Studienergebnisse mit Imatinib bei Patienten mit CML im fortgeschrittenen Stadium gaben einen Eindruck von der hohen Wirksamkeit und guten Verträglichkeit der Substanz. Dies führte dazu, dass die US-amerikanische Zulassungsbehörde FDA (Food and Drug Administration) bereits im Jahr 2001 einem beschleunigten Zulassungsantrag (fast-track approval) zustimmte, obwohl die Phase-II-Studien noch liefen. Parallel zum beschleunigten $\mathrm{Zu}$ lassungsantrag war eine große internationale, randomisierte Phase-III-Studie zum Erstlinieneinsatz von Imatinib bei nicht vorbehandelten CML-Patienten in der chronischen Phase gestartet worden (IRIS-Studie). Innerhalb der ersten 6 Monate erreichten im Imatinib-Arm $63 \%$ der Patienten eine gute zytogenetische Remission und weitere $40 \%$ eine komplette

\section{KARGER}

Fax +497614520714

Information@Karger.com

www.karger.com (c) 2013 S. Karger GmbH, Freiburg

0378-584X/13/3617-0002\$38.00/0

Accessible online at:

www.karger.com/onk 


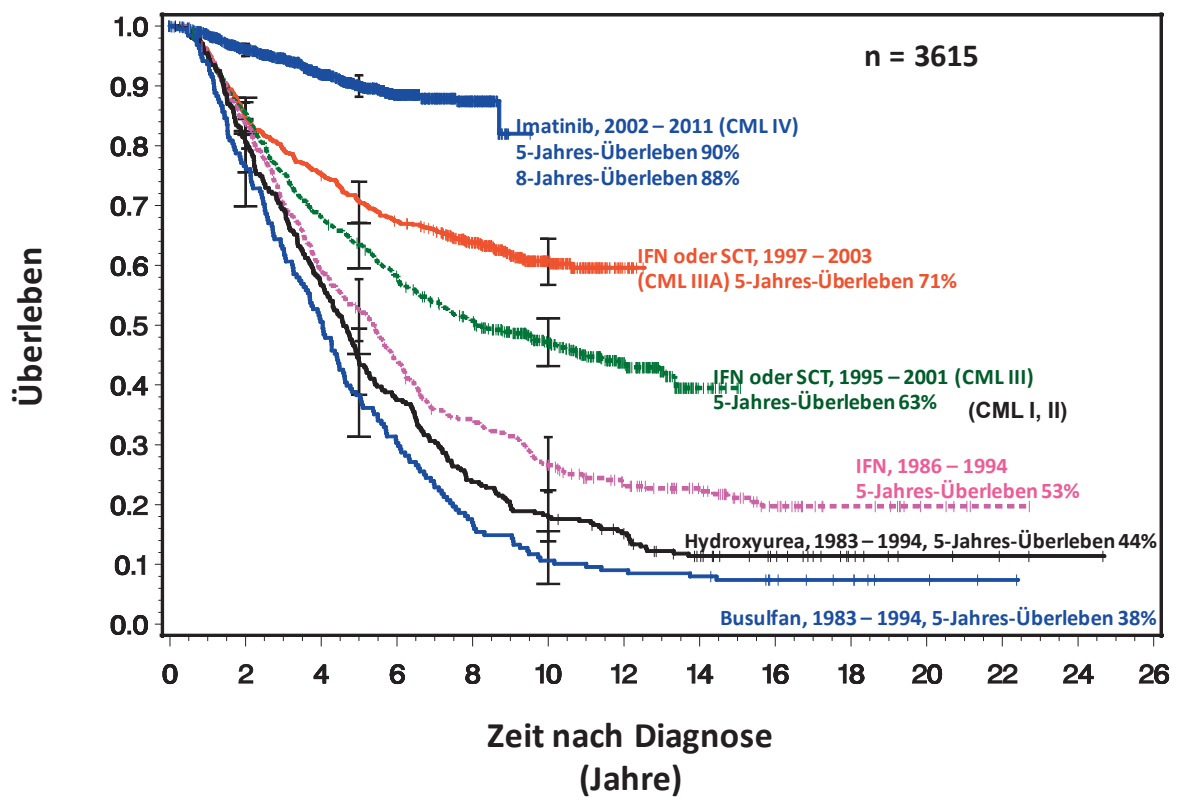

zytogenetische Remission im Vergleich zu 10\% bzw. 2\% der Patienten im Kontrollarm mit IFN/Cytarabin (Ara-C). Die Ergebnisse dieser Studie führten im Dezember 2002 zur Zulassung von Imatinib als Erstlinientherapie bei neu diagnostizierten CML-Patienten in der chronischen Phase.

Die mittlerweile vorliegenden Langzeitergebnisse bestätigen die anhaltend hohe Effektivität von Imatinib. Nach 5und 8-jähriger Nachbeobachtungszeit haben die mit Imatinib behandelten CML-Patienten ein deutlich verlängertes Gesamtüberleben im Vergleich zu Patienten, die mit IFN behandelt wurden (Abb. 1) [59].

In Deutschland erkranken pro Jahr etwa 1200 Menschen an einer CML. Basierend auf der zu erwartenden CML-Überlebenszeit ergibt sich für Europa für das Jahr 2050 eine CMLPrävalenz von 250 000, wobei die Diagnose bei der Mehrzahl der Betroffenen bereits viele Jahre zurückliegen wird. Das Ziel der medikamentösen Behandlung ist eine komplette zytogenetische und tiefe molekulare Remission, die auch nach Beendigung der Therapie lange anhält. Eine so definierte therapiefreie Remission (anhaltende Remission ohne Therapiebedarf) wird nur von einem Teil der Patienten erreicht und unterstreicht die Notwendigkeit eines fortlaufenden Monitorings. Eine Heilung der CML gelingt bisher in der Regel nur durch eine allogene Stammzelltransplantation.

Die CML-Therapie mit Imatinib und den zwischenzeitlich zugelassenen anderen Tyrosinkinaseinhibitoren wird fortlaufend optimiert. So wird bei Bcr-Abl-positiver CML ein rascher Therapiebeginn empfohlen, da nach Auswertung von Verlaufsdaten der IRIS-Studie eine frühe Reduktion der BcrAbl-Last (Bcr-Abl-Transkripte $\leq 0,1 \%$, gutes molekulares Ansprechen (MMR)) das Rezidivrisiko verringert und die Chance zur therapiefreien Remission erhöht [7].

\section{Offene Fragen zur Therapie der CML}

Aktuell geht es unter anderem darum, eine frühe molekulare Remission zu erkennen, um die Prognose der Patienten abschätzen zu können und dabei jene Patienten zu detektieren, die von einer frühen Stammzelltransplantation profitieren. Außerdem steht für Patienten, die innerhalb der initialen Therapiephase nicht ausreichend ansprechen, der Wechsel auf einen der neueren Tryrosinkinasehemmer (Nilotinib, Dasatinib) zur Diskussion. Hierzu gibt es bisher wegen fehlender Daten keine generellen Empfehlungen [8].

Die unbestreitbar großen Erfolge der Imatinib-Therapie bei Ph+ CML werden eingeschränkt durch die Resistenzproblematik. Neben molekulargenetischen Ursachen der Resistenzentwicklung (z.B. Punktmutationen oder Amplifikationen des Bcr-Abl-Gens) wurde als wesentlicher Grund für eine rasche Resistenzentwicklung eine mangelnde Therapiecompliance ausgemacht [9], die bei oralen Langzeittherapien ein generelles Problem ist. Des Weiteren wird die Resistenzentwicklung durch Polypragmasie gefördert. Bei Anwendung mehrerer Medikamente kann sich unter anderem die Absorptionsgeschwindigkeit ändern und das Risiko für Arzneimittelinteraktionen erhöhen. Eine britische Studie zeigt, dass mit Imatinib behandelte Patienten bei einer Therapieadhärenz von über $90 \%$ mit weitaus höherer Wahrscheinlichkeit ein MMR erreichen als Patienten mit schlechterer Therapieadhärenz [10]. Schon bald nach der Entwicklung von Imatinib wurden auch Mutationen beschrieben, die eine teilweise oder komplette Imatinib-Resistenz auslösen können [11]. Jedoch kann auch bei Patienten mit ImatinibResistenz das Protein CRKL-P, ein biochemischer Surrogatparameter für aktives Bcr-Abl, anhaltend niedrig sein [12]. Die Bcr-Abl-Aktivität wird bei diesen Patienten offenbar über einen alternativen Signalweg inhibiert. 
Abb. 2. ENESTnd-Studie: Imatinib versus Nilotinib in der Erstlinientherapie der CML (modifiziert nach [13]).

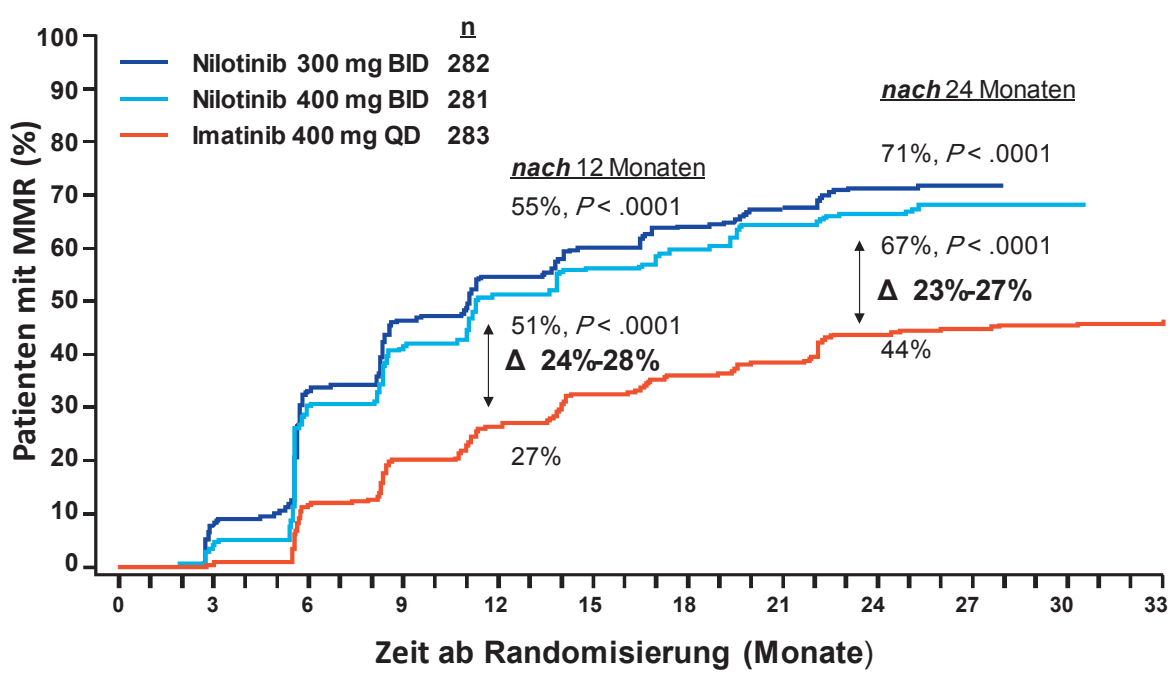

Tab. 1. Empfehlungen zu den Meilensteinen der CMLTherapie (modifiziert nach [14] und 15])

\begin{tabular}{llll}
\hline Meilenstein & Versagen & Warnsignal & Optimales Ansprechen \\
\hline Diagnose & & Hochrisiko, Major Route ACA & \\
3 Monate & keine $\mathrm{CHR}, \mathrm{Ph}>95 \%$ & $\mathrm{Ph}>35 \%, \mathrm{Bcr}-\mathrm{Abl}>10 \%$ & $\mathrm{Ph} \leq 35 \%, \mathrm{Bcr}-\mathrm{Abl} \leq 10 \%$ \\
6 Monate & $\mathrm{Ph}>35 \%, \mathrm{Bcr}-\mathrm{Abl}>10 \%$ & $\mathrm{Ph} 1-65 \%, \mathrm{Bcr}-\mathrm{Abl} 1-10 \%$ & $\mathrm{Ph} 0 \%, \mathrm{Bcr}-\mathrm{Abl} \leq 1 \%$ \\
12 Monate & $\mathrm{Ph} \geq 1 \%, \mathrm{Bcr}-\mathrm{Abl}>1 \%$ & $\mathrm{Bcr}-\mathrm{Abl} 0,1-1 \%$ & $\mathrm{Bcr}-\mathrm{Abl} \leq 0,1 \%$ \\
18-14 Monate & $\mathrm{Bcr}-\mathrm{Abl}>0,1 \%$ & $\mathrm{Bcr}-\mathrm{Abl} 0,01-0,1 \%$ & $\mathrm{Bcr}-\mathrm{Abl} \leq 0,01 \%$ \\
Jederzeit & Rezidiv, Verlust MMR & & \\
\hline
\end{tabular}

$\mathrm{CHR}=$ Komplette hämatologische Remission, $\mathrm{ACA}$ = zusätzliche zytogenetische Aberrationen.
Nach der Verankerung von Imatinib in der CML-Therapie wurden weitere Tyrosinkinaseinhibitoren entwickelt. Nilotinib wirkt Bcr-Abl-spezifischer, inhibiert vergleichbar mit Imatinib auch die Tyrosinkinasen PDGFR (platelet-derived growth factor receptor) und Kit und zeigt eine verbesserte zelluläre Bioverfügbarkeit. Dasatinib ist ein Multikinaseinhibitor mit Wirkung auf Abl, Src, PDGFR und Kit. Mit diesen Zweitgenerationsinhibitoren wurden schnellere und tiefere Remissionen im Vergleich zu Imatinib erreicht (Abb. 2), auch kann damit eine Imatinibresistenz bei etwa der Hälfte der davon betroffenen Patienten überwunden werden.

Das insgesamt sehr umfangreiche Datenmaterial zur CMLTherapie mit Tyrosinkinasehemmern wurde in Empfehlungen eingearbeitet, die die kritischen Interventionszeiträume («Meilensteine») benennen (Tab. 1). Die Empfehlungen finden sich in den Onkopedia-Leitlinien zur CML [14].

Noch offen ist die Frage, ob bei optimalem Vorgehen etwa entlang der Meilenstein-Tabelle die CML-Zellen vollständig eradiziert werden können. In vitro bleibt unter anhaltender Imatinib-Inkubation stets eine Restpopulation Bcr-Abl-positiver Zellen übrig, die aufgrund ihrer relativ geringen Bcr-Abl-Expression schlecht zugänglich für Imatinib sind [16]. Eine Depletion dieser Stammzellen dürfte folglich lange Zeit dauern. Derzeit ist die wichtigste Maßnahme für das Erreichen einer Therapiefreiheit die immunologische
Kontrolle, z.B. durch eine IFN-Therapie. Burchert et al. [17] beschreiben eine stabile Remission unter niedrigdosiertem IFN- $\alpha$ nach Absetzen von Imatinib. Die CML-V-Studie (TIGER-Studie) geht nun der Frage nach, ob eine Interferon-Erhaltungstherapie im Anschluss an Nilotinib/IFN die Prognose der Patienten nach Absetzen der Therapie weiter verbessert (Abb. 3).

Da die Tyrosinkinasen der PDGFR-Familie auch von anderen malignen Tumoren häufig überexprimiert werden, ergeben sich für Imatinib eine Reihe weiterer Einsatzgebiete. Dies sind insbesondere die GIST, die hypereosinophilen Syndrome/chronische Eosinophilenleukämie (CEL) und das Dermatofibrosarcoma protuberans (DFSP) mit Überexpression von PDGF, des Liganden des PDGFR. In folgenden Leitlinien der Deutschen Gesellschaft für Hämatologie und Medizinische Onkologie (DGHO) ist das jeweilige therapeutische Vorgehen beschrieben:

- Chronische Myeloische Leukämie (CML); Autoren: A. Hochhaus et al., Stand: 01/2013

- Chronische Myeloproliferative Erkrankungen; Autoren: E. Lengfelder et al., Stand: 04/2010

- Eosinophilie-assoziierte Myeloproliferative Erkrankungen (MPN-Eo); Autoren: A. Reiter et al., Stand: 09/2011

- Gastrointestinale Stromatumoren (GIST); Autoren: P. Reichardt, J. Schütte, Stand: 06/2011 


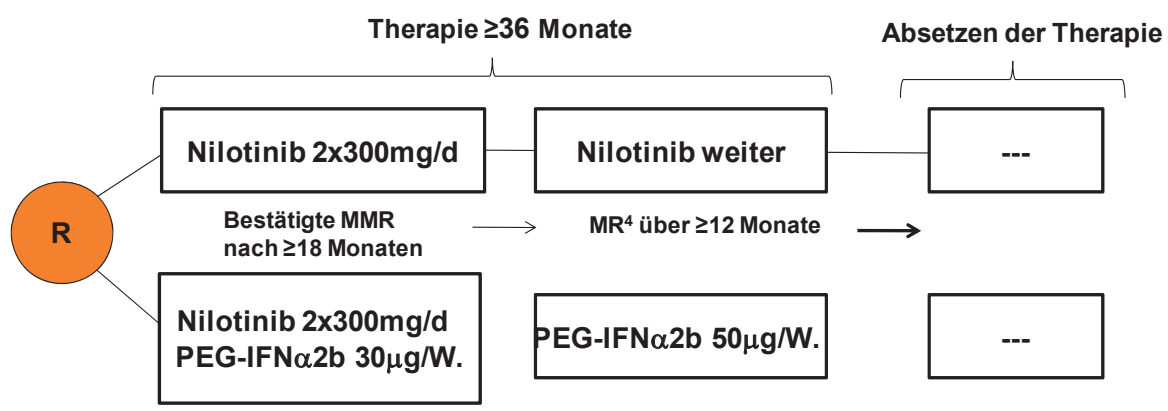

Abb. 3. Aufbau der CML-Studie V (TIGERStudie: $T \mathrm{KI}+I \mathrm{FN}$ trial initiated by the GERman CML Study Group) der Deutschen CML-Studiengruppe.

\section{Optimierte Induktion,}

deeskalierte Erhaltung,

Absetzen (Heilung?)

\section{Die Entwicklung gezielt wirkender Medikamente am Beispiel von Trastuzumab}

\section{Verantwortliche Autorin: Diana Lüftner, Berlin}

Trastuzumab (Herceptin ${ }^{\circledR}$, Roche Pharma AG, GrenzachWhylen, Deutschland) ist ein humanisierter monoklonaler Antikörper, der gezielt die Funktion des Proteins HER2 (humaner epidermaler Wachstumsfaktorrezeptor 2) hemmt. Aufgrund der in den zulassungsrelevanten Studien nachgewiesenen signifikanten Überlegenheit Trastuzumab-haltiger Kombinationstherapien wurde der Antikörper in Europa im Jahr 2000 zur Behandlung des metastasierten HER2-positiven Mammakarzinoms zugelassen. 2006 folgte die Zulassung für die adjuvante Therapiesituation bei Patientinnen mit HER2positivem Mammakarzinom im Frühstadium.

Drei Phase-III-Studien, die Trastuzumab beim metastasierten HER2-positiven Mammakarzinoms in der Monotherapie und kombiniert mit einem Taxan untersuchten, zeigten signifikante Verbesserungen der Ansprechraten, der Zeit bis zur Krankheitsprogression und des Gesamtüberlebens. In der randomisierten Phase-III-Zulassungsstudie H0648g wurde auch die Kombination Trastuzumab plus Standardchemotherapie (Doxorubicin oder Epirubicin plus Cyclophosphamid) als Erstlinientherapie bei HER2-positivem metastasiertem Mammakarzinom geprüft [18]. Die Anthrazyklin-haltigen Kombinationen waren zwar ebenfalls wirksamer als die Chemotherapie allein, wiesen aber eine erhöhte Kardiotoxizität auf. Daher wurde die Zulassung nur für die Kombination mit Paclitaxel beantragt. Tierexperimentelle Untersuchungen an Knockout-Mäusen bestätigten die Vermutung, dass die Blockade von ErbB2 (HER2/neu) den Herzmuskel schädigt, insbesondere in Kombination mit Anthrazyklinen [19].

Eine weitere Studie zum Einsatz von Trastuzumab beim metastasierten Mammakarzinom war von untergeordnetem wissenschaftlichem Interesse, da sie in einem vergleichbaren Setting ein weiteres Taxan (Docetaxel) in Kombination mit
Trastuzumab prüfte [20]. Im Ergebnis zeigte sich ebenfalls eine signifikante Erhöhung der progressionsfreien Zeit, des Gesamtüberlebens, der Ansprechraten und der Ansprechdauer bei Hinzunahme von Trastuzumab. Auffällig war in dieser im Cross-over-Design angelegten Studie, dass der gänzliche Verzicht auf Trastuzumab (Frauen im Docetaxel-Arm ohne Cross-over) mit einer verkürzten Überlebenszeit korrelierte. Die Hinzunahme von Trastuzumab im Falle der Progression (Frauen im Doxetaxel-Arm mit Cross-over) führte demgegenüber zu einem Überlebensvorteil, der allerdings geringer ausfiel als bei Frauen, die von Anfang an mit Trastuzumab plus Docetaxel behandelt worden waren (Abb. 4). Dies gilt als Beleg dafür, dass bei Nachweis einer HER2Überexpression möglichst ohne Zeitverzug mit der Trastuzumab-Therapie begonnen werden sollte.

Verschiedene Studien zur Kombination von Trastuzumab/ Taxan mit Platinsalzen zeigten zwar, dass sie das progressionsfreie Überleben der Patientinnen gegenüber der Therapie mit Trastuzumab/Taxan weiter verlängern können, solche Dreifachkombinationen sind jedoch aktuell nicht zugelassen.

\section{Trastuzumab in der adjuvanten Therapie}

In der adjuvanten Situation wurde der Einsatz von Trastuzumab beim HER2-positiven Mammakarzinom in unterschiedlichen Regimes und Dosierungsschemata in 4 großen multizentrischen Studien in den USA und Europa erfolgreich untersucht (HERA, BCIRG 006, NSABP-B31/NCCTGN9831) [21-26]. Primärer Studienendpunkt war das krankheitsfreie Überleben (DFS), das in allen Studien signifikant zugunsten der Trastuzumab-haltigen Therapieprotokolle ausfiel. Auch im medianen Gesamtüberleben schnitten die Trastuzumab-Gruppen besser ab, wobei in der langjährigen Nachbeobachtung die Unterschiede $\mathrm{zu}$ den Kontrollgruppen wegen der Möglichkeit zum Cross-over (Hinzunahme von Trastuzumab im Falle eines Rezidivs) geringer wurden.

An keiner der Studien zur adjuvanten Therapie mit Trastuzumab bei HER2-Überexpression haben Frauen mit kleinen Tumoren (T1) teilgenommen. Retrospektive Analysen von 
Abb. 4. Kombination von Trastuzumab und Docetaxel (modifiziert nach [20]).

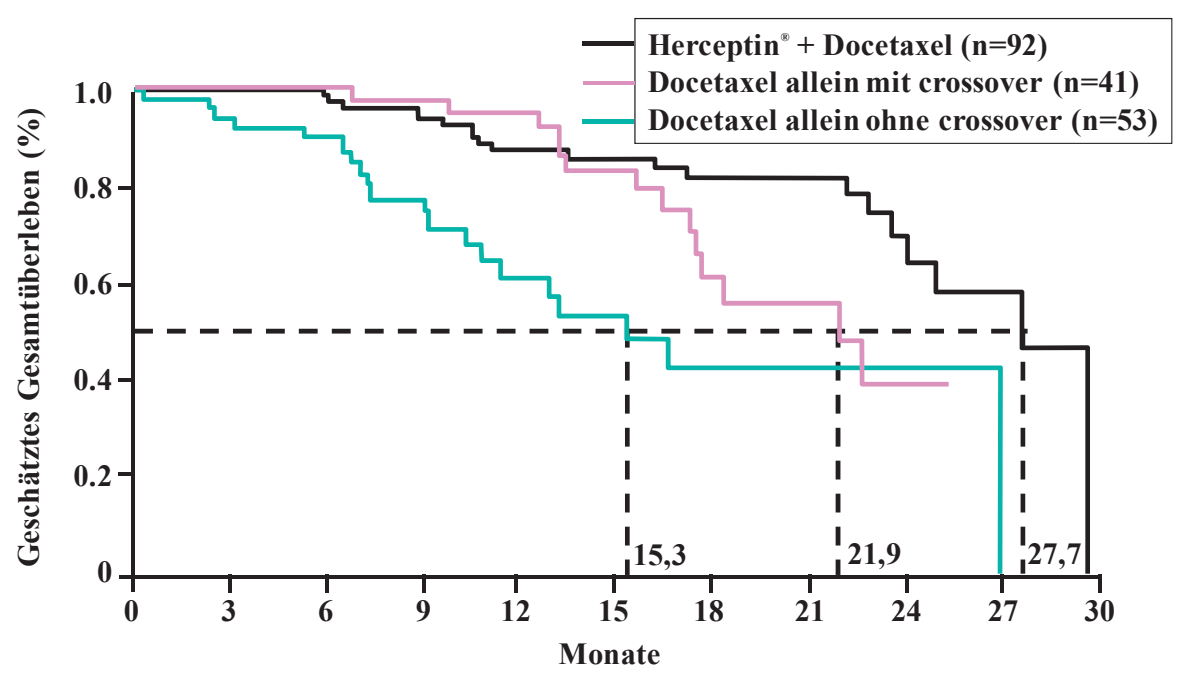

Patientendaten amerikanischer Kliniken zeigten jedoch, dass die Prognose von kleinen, HER2-positiven Tumoren schlechter ist als die HER2-negativer Tumoren und sogar noch ungünstiger als die der tripel-negativen Tumoren. Deshalb empfehlen amerikanische Leitlinien [27] die Therapie mit Trastuzumab auch bei kleinen Tumoren $(0,6-1 \mathrm{~cm})$, obwohl entsprechende Studien fehlen.

Eine ebenfalls retrospektive Analyse aus dem umfangreichen Datenmaterial amerikanischer Krebszentren ging der Frage nach, ob Patientinnen mit adjuvanter Trastuzumab-Therapie im Falle eines Wiederauftretens der Erkrankung erneut von einer Behandlung mit dem Antikörper profitieren [28]. Analysiert wurde eine Gruppe von 164 Patientinnen (aus einer Kohorte von 2049 Patientinnen), die im Anschluss an eine adjuvante Trastuzumab-Therapie nach durchschnittlich 5 Monaten ein systemisches Tumorrezidiv hatten. Die Daten zeigen, dass Frauen mit erneuter Trastuzumab-Therapie zusätzlich zur Chemotherapie $(n=122)$ ein längeres progressionsfreies Überleben hatten als Frauen, die nur eine Chemotherapie erhielten $(n=42)$. Patientinnen mit adjuvanter TrastuzumabTherapie profitieren demnach in der metastasierten Situation durchaus von einer Re-Therapie mit dem Antikörper.

\section{Die adjuvante Therapie mit Trastuzumab: zur Frage der Dauer}

Nach jetzt mehr als 10-jähriger Erfahrung mit Trastuzumab stellt sich - nicht zuletzt mit Blick auf die hohen Kosten dieser Behandlung - die Frage nach einer möglichen Änderung der Therapiedauer. Zur Diskussion steht sowohl eine Verkürzung der Therapie mit dem Ziel einer verringerten Nebenwirkungsrate bei gleichzeitiger Kostenersparnis als auch die Verlängerung der Therapie auf z.B. 2 Jahre mit dem Ziel eines noch größeren Benefits für die Patientinnen.

In der auf Nichtunterlegenheit angelegten Studie PHARE wurde Trastuzumab entweder für 6 oder für 12 Monate gegeben [29]. PHARE berücksichtigte Patientinnen mit frühem,
HER2-positivem Brustkrebs, die mindestens 4 Zyklen Chemotherapie erhalten hatten. Nach 6-monatiger adjuvanter Trastuzumab-Behandlung erfolgte die Randomisierung entweder in den Standardtherapie-Studienarm mit fortgesetzter Verabreichung von Trastuzumab für weitere 6 Monate oder in die zweite Gruppe, bei der Trastuzumab abgesetzt wurde. Die Nichtunterlegenheit der 6-monatigen Trastuzumab-Behandlung galt als nachgewiesen und damit der primäre Studienendpunkt als erreicht, wenn die absolute Verringerung des DFS im Vergleich zur 12-monatigen Behandlung 2\% betrug. Daraus leitete sich der relative Grenzwert der Hazard Ratio (HR) für eine Nichtunterlegenheit ab: Dieser lag bei 1,15 für das 95\%-Konfidenzintervall des zu ermittelnden DFS. Nach median 42,5 Monaten waren 10,4\% der Patientinnen des Standardtherapie-Studienarms (12 Monate Trastuzumab) von einem DFS-Ereignis betroffen, in der experimentellen Gruppe (6 Monate Trastuzumab) waren es 13,0\%. Die HR bezüglich des DFS lag nichtsignifikant bei 1,28, was einem um $28 \%$ erhöhtem Risiko eines Rezidivs oder Tod aufgrund der verkürzten Trastuzumab-Therapie entspricht. Obgleich die 6-Monats-Therapie eine geringere Kardiotoxizität hatte, kann eine Unterlegenheit gegenüber der 12-Monats-Therapie demnach nicht ausgeschlossen werden. Zwar waren die Unterschiede im progressionsfreien Überleben und im Gesamtüberleben nicht sehr groß, trotzdem kann eine Verkürzung der Behandlung mit Trastuzumab auf Grundlage dieser Studie nicht empfohlen werden.

Zur Frage, ob eine längere Therapie mit Trastuzumab die Ergebnisse weiter verbessert, kann die finale Auswertung der HERA-Studie herangezogen werden [30]. Eingeschlossen in die Untersuchung wurden mehr als 5000 Patientinnen mit HER2-positivem frühen Brustkrebs, die nach Operation und (neo)adjuvanter Chemotherapie ( $\geq 4$ Zyklen) und gegebenenfalls zusätzlicher Radiotherapie in 3 Studienarme randomisiert wurden: eine Beobachtungsgruppe ohne Anti-HER2Therapie, eine Gruppe mit 1 Jahr Trastuzumab-Behandlung 
Abb. 5. Progressionsfreies Überleben unter T-DM1 im Vergleich zur Standardtherapie (modifiziert nach [34]).

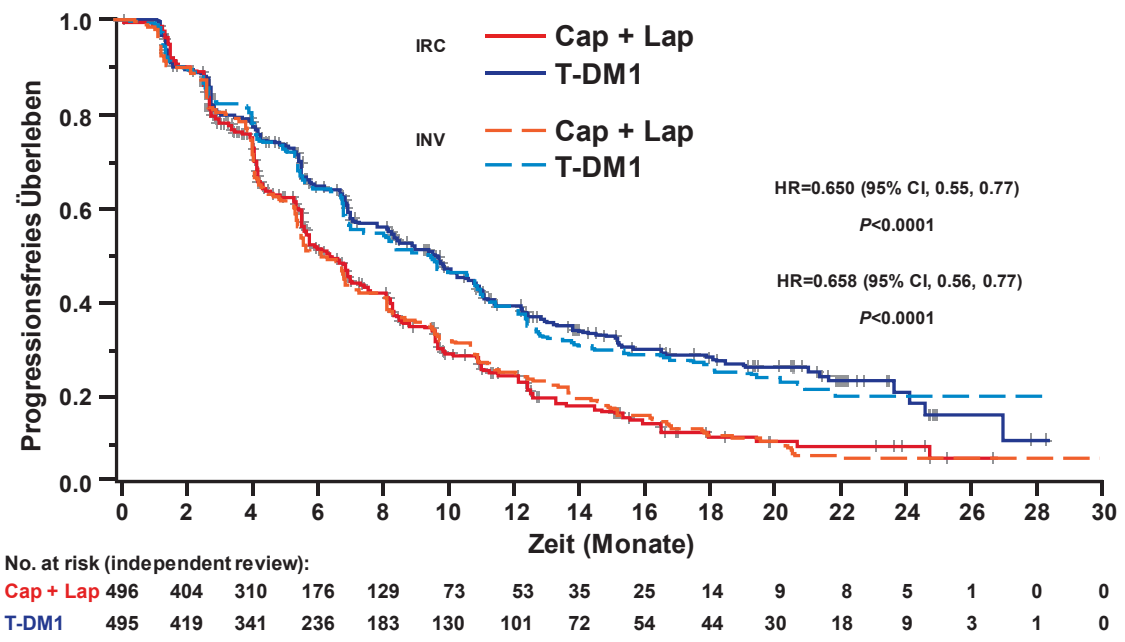

und eine Gruppe mit 2-jähriger Trastuzumab-Behandlung. Das DFS war in beiden Trastuzumab-Studienarmen (2 Jahre vs. 1 Jahr) praktisch gleich und lag nach 8 Jahren Nachbeobachtung bei 75,8\% bzw. 76,0\% (HR 0,99; p = 0,86). Der Hormonrezeptorstatus hatte keinen Einfluss auf das DFS. Auch hinsichtlich des Gesamtüberlebens unterschieden sich die beiden mit Trastuzumab behandelten Gruppen nicht signifikant. Es bleibt somit vorerst bei der 1-jährigen TrastuzumabTherapie, zumal in der HERA-Studie die Verlängerung der Therapie auf 2 Jahre mit einer erhöhten Kardiotoxizität verbunden war.

\section{Alternativen zu Trastuzumab?}

Auf dem letztjährigen American Society of Clinical Oncology (ASCO)-Meeting wurde eine 2-armige Studie vorgestellt, die bei Patientinnen mit HER2-positivem, metastasiertem Mammakarzinom die Erstlinientherapie mit Trastuzumab/Taxan einer Therapie mit Lapatinib/Taxan gegenübergestellt hat [31]. Das in dieser Indikation nicht zugelassene Lapatinib war dem Trastuzumab deutlich unterlegen in Bezug auf die Überlebensendpunkte, auch war in der LapatinibGruppe die Toxizität höher. Die Kombination von Lapatinib und Trastuzumab könnte jedoch eine Option für mit Trastuzumab vorbehandelte und danach progredient gewordene $\mathrm{Pa}$ tientinnen sein. Offenbar kann damit eine Trastuzumab-Resistenz teilweise überwunden werden, da in einer Studie ein Überlebensvorteil der Dublette gegenüber der Lapatinib-Monotherapie nachgewiesen wurde [32].

\section{Wie geht es weiter?}

Mit Pertuzumab und dem Fusionsprodukt T-DM1 (Trastuzumab gekoppelt mit dem Spindelgift DM1) sind 2 neue Substanzen in der fortgeschrittenen klinischen Entwicklung bzw. bereits verfügbar (Pertuzumab), die die Wirksamkeit von Trastuzumab offenbar verstärken können. Dies zeigte sich zunächst in der CLEOPATRA-Studie [33], an der Frauen mit
HER2-positivem, metastasiertem Brustkrebs teilgenommen haben. Sie erhielten entweder eine Kombination von Pertuzumab und Trastuzumab plus Docetaxel oder nur Trastuzumab und Docetaxel. Die Resultate zeigen, dass bei Patientinnen, die mit Pertuzumab behandelt wurden, das progressionsfreie Überleben signifikant länger war (18,7 vs. 12,4 Monate; HR $=0,69$ ) als bei Patientinnen, die kein Pertuzumab erhielten. Für Patientinnen unter 75 Jahren konnte auch ein Überlebensvorteil nachgewiesen werden. Dass die duale Blockade von HER2 weniger kardiale Schäden als die Behandlung mit Trastuzumab allein verursacht, wie sich aus der Studie ebenfalls entnehmen lässt, bedarf noch der weiteren Klärung.

Eine weitere Phase-III-Studie ist EMILIA, die das Konjugat Trastuzumab-Emtansin (T-DM1) mit Lapatinib plus Capecitabin vergleicht [34]. Sie schließt Patientinnen mit HER2positivem inoperablem, lokal fortgeschrittenem oder metastasiertem Mammakarzinom nach vorangegangener Therapie mit Trastuzumab plus Taxan ein. T-DM1 hat nach dem derzeitigen Stand (Daten zum Gesamtüberleben stehen noch aus) einen hohen therapeutischen Index: Es verlängert das progressionsfreie Überleben (Abb. 5) bei offenbar sehr guter Verträglichkeit.

Auch in der neoadjuvanten Therapie bei frühem HER2positivem Brustkrebs könnten Anti-HER2-Dubletten künftig eine Rolle spielen. So hat die NEOSPHERE-Studie [35] gezeigt, dass die neoadjuvante Therapie mit Pertuzumab plus Trastuzumab plus Docetaxel die Wahrscheinlichkeit eines kompletten pathologischen Ansprechens signifikant um mehr als die Hälfte verbessert verglichen mit Trastuzumab plus Docetaxel (45,8 vs. $29,0 \%$; $p=0,014)$.

Überdies dürfte künftig die subkutane Applikation von Trastuzumab möglich sein, nachdem in einer Studie die Nichtunterlegenheit der subkutanen Injektion gegenüber der intravenösen Verabreichung gezeigt worden ist [36]. Dies wird die AntiHER2-Therapie für die Patientinnen erheblich vereinfachen. 
Tab. 2. Schema zur Behandlung bei metastasiertem Mammakarzinom

\begin{tabular}{lll}
\hline & Standard & Gute Datenlage oder ausstehende Zulassung \\
\hline Erstlinie & Taxane + Trastuzumab & Taxane + Trastuzumab (iv und sc) + Pertuzumab \\
Zweitlinie & Lapatinib + Capecitabin & TDM-1 \\
& (treatment beyond progression oft geübt) & Lapatinib + Capecitabin \\
Folgelinien & $($ Lapatinib + Trastuzumab), & Lapatinib + Capecitabin, \\
& $($ andere Chemotherapie + Trastuzumab) & Lapatinib + Trastuzumab (iv und sc) \\
\hline
\end{tabular}

iv= Intravenös, $\mathrm{sc}=$ subkutan.
Auf Basis der mittlerweile sehr umfangreichen Datenlage zu Trastuzumab wird der Antikörper bei metastasiertem Mammakarzinom in nahezu allen Therapielinien eingesetzt (Tab. 2).

Studien mit neuen Substanzen werden das derzeitige Vorgehen schrittweise verändern. Das gilt auch für die Therapie bei neu diagnostiziertem Brustkrebs. So prüft die MARIANNEStudie die Wirksamkeit und Sicherheit von Trastuzumab-Emtansin (T-DM1) in Kombination mit Pertuzumab bzw. Placebo versus einer Kombinationstherapie mit Trastuzumab und einem Taxan (Docetaxel oder Paclitaxel) bei Patientinnen mit HER2-positivem, fortgeschrittenem oder lokal rezidivierend fortgeschrittenem oder bisher unbehandeltem metastasiertem Brustkrebs [37]. Die Ergebnisse dieser Studie, deren Rekrutierung bereits abgeschlossen ist, werden mit Spannung erwartet.

\section{Das Konzept der zielgerichteten Tumor- Immuntherapie}

\section{Verantwortlicher Autor: Andreas Mackensen, Erlangen}

Es gibt zahlreiche Belege, dass es bei Tumorerkrankungen zu Abwehrreaktionen durch körpereigene immunkompetente Zellen kommt. Beispielhaft sei hier der Graft-versus-Leukämie-Effekt nach allogener Stammzelltransplantation genannt, der den wesentlichen Wirkungsmechanismus für den therapeutischen Erfolg dieser Therapiemodalität darstellt [38]. Auch bei anderen malignen Tumoren konnte eine spezifische Immunantwort durch T-Zellen, die tumorassoziierte Antigene (TAA) erkennen, nachgewiesen werden [39]. Dabei scheint die Dichte der T-Lymphozyten im primären Tumorgewebe mit einer besseren Prognose zu korrelieren, wie Untersuchungen beim kolorektalen Karzinom gezeigt haben [40]. Ferner konnte der Nachweis erbracht werden, dass klonal expandierte, TAA-spezifische T-Zellen für die seltene Spontanregression von malignen Melanomen verantwortlich sind [41]. In der Regel gelingt es dem Immunsystem jedoch trotz hoher Aktivität nicht, das Tumorwachstum effektiv und langfristig zu kontrollieren. Tumorzellen haben zahlreiche Escape-Mechanismen entwickelt, um sich gegen den Angriff immunkompetenter Zellen zu schützen [42]. Bei der Entwicklung von Immuntherapien ist die Überwindung dieser EscapeMechanismen von zentraler Bedeutung.

\section{Gezielte Immuntherapie mit Impfstoffen}

Alle therapeutischen Impfstoffe gegen maligne Tumore befinden sich noch in der Entwicklung und sollten deshalb nur im Rahmen von kontrollierten klinischen Studien angewendet werden. Das Problem der therapeutischen Tumorvakzinierung besteht darin, dass die Ignoranz bzw. Toleranz der Immunzellen gegen Tumorzellantigene nur schwer zu durchbrechen ist, zumal sich die Tumorzellen bei manifester Erkrankung der Immunüberwachung weitgehend entzogen haben.

Die aktive therapeutische Vakzinierung verfolgt den Ansatz, durch subkutane oder intramuskuläre Injektion spezifischer TAA die Aktivierung immunkompetenter TAA-spezifscher Immunzellen im Lymphgewebe anzukurbeln [43]. Mittlerweile konnten zahlreiche TAA identifiziert werden, die sich prinzipiell für die Entwicklung von Impfstoffen eignen. Ein vielversprechender Impfstoff-Kandidat ist die MAGEA3-Vakzine, die bei Patienten mit nichtkleinzelligem Lungenkarzinom (NSCLC; Stadium IB/II) zunächst erfolgreich im Rahmen einer Phase-II-Studie geprüft wurde [7]. Patienten, die mit dem MAGE-A3-Impfstoff behandelt wurden, hatten nach chirurgischer Tumorentfernung im Vergleich zu Placebo ein zwar nicht signifikantes, aber doch um $27 \%$ vermindertes relatives Risiko für ein Tumorrezidiv (Abb. 6).

Daraufhin wurde eine Phase-III-Studie mit dem Krebsimpfstoff aufgelegt, bei der die MAGE-A3-Vakzine als adjuvante Therapie bei rund 2200 MAGE-A3-positiven Patienten mit operativ vollständig entferntem NSCLC der Stadien IB, II und IIIA geprüft wird. Die Verabreichung des MAGE-A3Impfstoffs erfolgt in 2 Patientengruppen: in einer Gruppe nach Operation und Standardchemotherapie, in der anderen Gruppe nach Operation ohne zusätzliche Chemotherapie. Der primäre Endpunkt der Studie ist die krankheitsfreie Überlebenszeit.

Ebenfalls in der fortgeschrittenen klinischen Entwicklung ist eine Multipeptid-Vakzine beim metastasierten Nierenzellkarzinom, die zusammen mit dem Immunmodulator GM-CSF (Granulozyten/Makrophagen-Kolonien stimulierender Faktor) und niedrigdosiertem Cyclophosphamid (Letzteres mit dem Ziel der Lymphodepletion) appliziert wird. Im Rahmen einer Phase-II-Studie hatte sich die Vakzine als sicher erwiesen. Eine durch sie induzierte Immunantwort war mit einem signifikanten Überlebensvorteil verbunden. Die positiven Studienergebnisse waren auch in diesem Fall ausschlaggebend 
Abb. 6. Verlängertes DFS nach MAGE-A3Vakzinierung (modifiziert nach [44]).

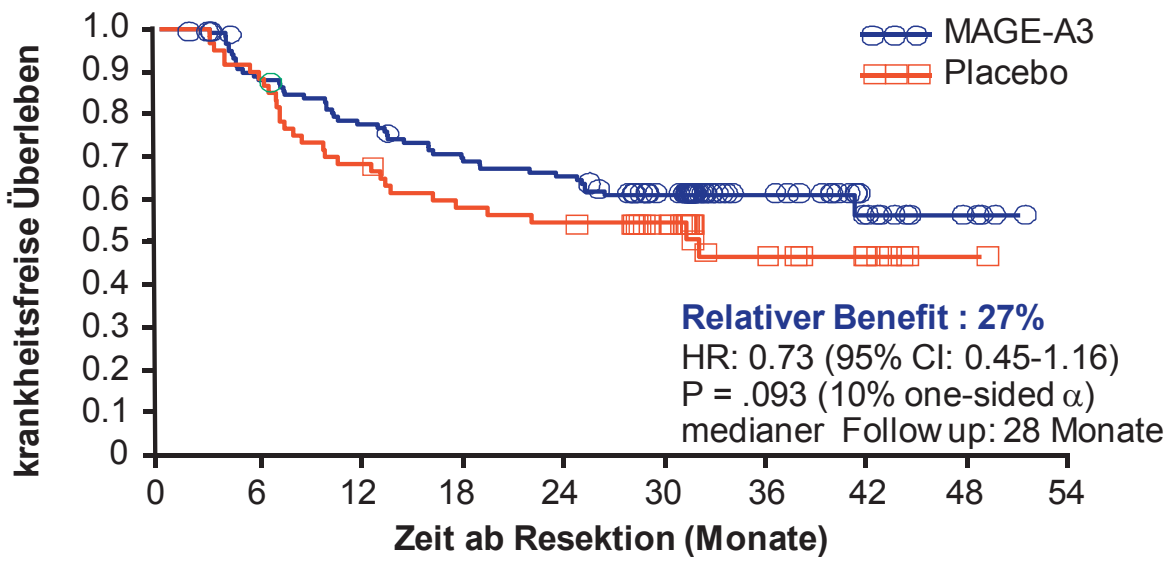

gp100; nach 2 Jahren waren es $24 \%$ versus $14 \%$. $\mathrm{Zu}$ beachten ist, dass einige Patienten erst mit Verzögerung (und nach zwischenzeitlichem Progress) auf die Immuntherapie angesprochen haben. Aus der Studie lässt sich weiterhin entnehmen, dass ein kleinerer Teil der Patienten auch langfristig (bis zu 6 Jahre) von der Behandlung profitiert und dass auch Patienten mit Hirnmetastasen auf eine solche Therapie ansprechen [48, 49].

Die Therapie mit Ipilimumab hatte zum Teil ausgeprägte Nebenwirkungen im Zusammenhang mit einer überschießenden Immunreaktion, dazu gehörten Darmentzündungen und Hautreaktionen. Es kam auch zu Entzündungen der Hypophyse [50, 51]. 10-15\% der Patienten in der Antikörper-Gruppe hatten schwerwiegendere immunologische Nebenwirkungen von Grad 3 oder 4 gegenüber 3\% in der gp100-Gruppe.

Noch in klinischer Entwicklung ist ein Antikörper, der an das ko-inhibitorische Molekül PD-1 bindet. Es wird auf TZellen exprimiert, bremst ebenfalls die T-Zellaktivierung nach Antigenstimulation und löst den programmierten Zelltod aus. Durch Blockade von PD-1 könnte zusätzlich der molekulare Schutzschild der Tumorzellen gegen endogene Abwehrmaßnahmen aufgebrochen werden [52]. In einer Phase-IStudie mit fast 300 Patienten wurden mit einem Anti-PD-1Antikörper relativ hohe Ansprechraten erzielt: Nach 6-monatiger Therapie waren $28 \%$ der teilnehmenden Melanompatienten in kompletter oder partieller Remission, $18 \%$ Prozent der Patienten mit NSCLC und 27\% der Patienten mit Nierenzellkarzinom [53]. Bei zwei Dritteln der Patienten mit objektivem Ansprechen hielt dieses für mindestens 12 Monate an. Alle Patienten hatten eine fortgeschrittene Erkrankung und waren vorbehandelt. Patienten, in deren Tumorgewebe der korrespondierende Ligand PD-L1 nachgewiesen werden konnte, sprachen auf die Antikörper-Therapie am besten an, sodass sich PD-L1 womöglich als Biomarker für das Tumoransprechen eignet.

Trotz der nur langsamen Fortschritte ist die Immuntherapie mit großen Erwartungen verknüpft und wird als eines der Therapiekonzepte mit hohem Potenzial für die Krebsbekämpfung angesehen [54]. 


\section{Adoptiver Immunzelltransfer}

Das Konzept der adoptiven zellulären Immuntherapie gegen Tumoren verfolgt das Ziel der Elimination bösartiger Zellen durch die Rückübertragung zuvor entnommener und dann modifizierter T-Lymphozyten eines Krebspatienten [55]. Dafür werden entweder die Immunzellen des Patienten mit einem transgenen T-Zell-Rezeptor spezifisch für TAA oder mit chimären, für Tumoroberflächenantigene spezifischen Rezeptorstrukturen gentechnisch gekoppelt [56]. Mit T-Zellen, die über einen viralen Vektor mit einem zweiten T-Zell-Rezeptor für das TAA NY-ESO-1 versehen wurden, wurden in Pilotstudien Remissionen bei synovialen Sarkomen und bei Melanomen erzielt [57]. Die Immuntherapie unter Verwendung chimärer Antigenrezeptoren wird derzeit in Kooperation mit der pharmazeutischen Industrie intensiv vorangetrieben, nachdem mit diesem Ansatz unter Verwen- dung eines gegen CD19 gerichteten Antigenrezeptors gute Ansprechraten bei Patienten mit chronischer lymphatischer Leukämie (CLL) erzielt worden waren [58]. Der adoptive Immunzelltransfer ist somit ein vielversprechender Ansatz, wobei es Hinweise gibt, dass die Effektivität durch weitere Maßnahmen (z.B. eine vorausgehende Lymphozyten depletierende Chemotherapie) noch weiter verbessert werden kann.

\section{Disclosure Statement}

A.H. erhielt Forschungsunterstützung von Novartis, Bristol-Myers Squibb, Ariad, Pfizer, MSD. D.L. erhielt Vortragshonorare von Hoffmann-LaRoche und GSK. A.M. erhielt Vortragshonorare von BristolMyers Squibb.

\section{Literatur}

1 Nowell PC, Hungerford DA: A minute chromosome in human granulocytic leukemia. Science 1960;132:1497.

2 Abelson HT, Rabstein LS: Lymphosarcoma: virusinduced thymic-independent disease in mice. Can cer Res 1970;30:2213-2222.

-3 Rosenberg $\mathrm{N}$ et al.: Characterization of Abelson murine leukemia virus isolates differing in P120 expression. Cold Spring Harb Symp Quant Biol 1980; 44(Pt 2):859-864.

4 Daley GQ et al.: Induction of chronic myelogenous leukemia in mice by the P210bcr/abl gene of the Philadelphia chromosome. Science 1990;247:824830.

5 Heisterkamp $\mathrm{N}$ et al.: Acute leukaemia in bcr/abl transgenic mice. Nature 1990;344:251-223.

6 Bonifazi $\mathrm{F}$ et al.: Chronic myeloid leukemia and interferon-alpha: a study of complete cytogenetic responders. Blood 2001;98:3074-3081.

$>7$ Hughes TP et al.: Long-term prognostic significance of early molecular response to imatinib in newly diagnosed chronic myeloid leukemia: an analysis from the International Randomized Study of Interferon and STI571 (IRIS). Blood 2010;116: 3758-3765.

8 Hanfstein B et al.: Early molecular and cytogenetic response is predictive for long-term progressionfree and overall survival in chronic myeloid leukemia (CML). Leukemia 2012;26:2096-2102.

9 Apperley JF: Part I: Mechanisms of resistance to imatinib in chronic myeloid leukaemia. Lancet Oncol 2007;8:1018-1029.

10 Marin D et al.: Adherence is the critical factor for achieving molecular responses in patients with chronic myeloid leukemia who achieve complete cytogenetic responses on imatinib. J Clin Oncol 2010;28:2381-2388.

11 Corbin AS et al.: Several Bcr-Abl kinase domain mutants associated with imatinib mesylate resistance remain sensitive to imatinib. Blood 2003;101: 4611-4614.

12 Hochhaus A et al.: Molecular and chromosomal mechanisms of resistance to imatinib (STI571) therapy. Leukemia 2002;16:2190-2196.

13 Saglio G et al.: Nilotinib versus imatinib for newly diagnosed chronic myeloid leukemia. N Engl J Med 2010;362:2251-2259.
14 www.dgho-onkopedia.de/de/onkopedia/leitlinien/cml.

15 Hochhaus A et al.: Chronische myeloische Leukämie. Heilung durch medikamentöse Therapie? Onkologe 2012;18:1105-1114.

16 Graham SM et al.: Primitive, quiescent, Philadelphia-positive stem cells from patients with chronic myeloid leukemia are insensitive to STI571 in vitro. Blood 2002;99:319-325.

17 Burchert A et al.: Sustained molecular response with interferon alfa maintenance after induction therapy with imatinib plus interferon alfa in patients with chronic myeloid leukemia. J Clin Oncol 2010;28:1429-1435.

18 Slamon DJ et al.: Use of chemotherapy plus a monoclonal antibody against HER2 for metastatic breast cancer that overexpresses HER2. N Engl J Med 2001;344:783-792.

19 Crone SA et al.: ErbB2 is essential in the prevention of dilated cardiomyopathy. Nat Med 2002;8: 459-465.

20 Marty $\mathrm{M}$ et al.: Randomized phase II trial of the efficacy and safety of trastuzumab combined with docetaxel in patients with human epidermal growth factor receptor 2-positive metastatic breast cancer administered as first-line treatment: the M77001 study group. J Clin Oncol 2005;23:4265-4274.

21 Piccart-Gebhart MJ et al.: Trastuzumab after adjuvant chemotherapy in HER2-positive breast cancer. N Engl J Med 2005;353:1659-1672.

22 Smith I et al.: 2-Year follow-up of trastuzumab after adjuvant chemotherapy in HER2-positive breast cancer: a randomised controlled trial. Lancet 2007;369:29-36.

23 Gianni L et al.: Treatment with trastuzumab for 1 year after adjuvant chemotherapy in patients with HER2-positive early breast cancer: a 4-year followup of a randomised controlled trial. Lancet Oncol 2011;12:236-244.

24 Slamon D et al.: Adjuvant trastuzumab in HER2positive breast cancer. N Engl J Med 2011;365: 1273-1283.

25 Romond EH et al.: Trastuzumab plus adjuvant chemotherapy for operable HER2-positive breast cancer. N Engl J Med 2005;353:1673-1684.
26 Perez EA et al.: Four-year follow-up of trastuzumab plus adjuvant chemotherapy for operable human epidermal growth factor receptor 2-positive breast cancer: joint analysis of data from NCCTG N9831 and NSABP B-31. J Clin Oncol 2011;29: 3366-3373.

27 www.nccn.org.

28 Gruschkus SK et al.: First-line patterns of care and outcomes of HER2-positive breast cancer patients who progressed after receiving adjuvant trastuzumab in the outpatient community setting. J Clin Oncol 2010;28(suppl 15):abstr 684.

29 Pivot X et al.: PHARE trial results comparing 6 to 12 months of trastuzumab in adjuvant early breast cancer. European Society for Medical Oncology (ESMO) 2012;abstr LBA5_PR and oral presentation

30 Goldhirsch A et al.: HERA trial: 2 years versus 1 year of trastuzumab after adjuvant chemotherapy in women with HER2-positive early breast cancer at 8 years of median follow up. European Society for Medical Oncology (ESMO) 2012; abstr LBA6_ $\mathrm{PR}$ and oral presentation.

31 Gelmon K et al.: Open-label phase III randomized controlled trial comparing taxane-based chemotherapy (Tax) with lapatinib (L) or trastuzumab (T) as first-line therapy for women with HER2+ metastatic breast cancer: interim analysis (IA) of NCIC CTG MA.31/GSK EGF 108919. American Society of Clinical Oncology (ASCO) Annual Meeting 2012; abstr LBA671.

32 Blackwell KL et al.: Updated survival analysis of a randomized study of lapatinib alone or in combination with trastuzumab in women with HER2-positive metastatic breast cancer progressing on trastuzumab therapy. San Antonio Breast Cancer Symposium (SABCS) 2009; abstr 61.

33 Baselga J et al.: Pertuzumab plus trastuzumab plus docetaxel for metastatic breast cancer. N Engl J Med 2012:366:109-119.

34 Blackwell KL et al.: Primary results from EMILIA, a phase III study of trastuzumab emtansine (TDM1) versus capecitabine (X) and lapatinib (L) in HER2-positive locally advanced or metastatic breast cancer (MBC) previously treated with trastuzumab (T) and a taxane. J Clin Oncol 2012;30 (suppl):abstr LBA1. 
35 Gianni L et al.: Neoadjuvant pertuzumab (P) and trastuzumab $(\mathrm{H})$ : antitumor and safety analysis of a randomized phase II study (,NeoSphere'). San Antonio Breast Cancer Symposium (SABCS) 2010; abstr S 3-2.

36 Ismael G et al.: Subcutaneous versus intravenous administration of (neo)adjuvant trastuzumab in patients with HER2-positive, clinical stage I-III breast cancer (HannaH study): a phase 3, open-label, multicentre, randomised trial. Lancet Oncol 2012;13:869-878.

37 Perez E et al.: Efficacy and safety of trastuzumabDM1 vs trastuzumab plus docetaxel in HER2-positive metastatic breast cancer patients with no prior chemotherapy for metastatic disease: Preliminary results of a randomized, multicenter, open-labe phase 2 study (TDM4450G). Proceedings European Society for Medical Oncology (ESMO) 2010; abstr LBA3.

38 Kolb HJ et al.: Donor leukocyte transfusions for treatment of recurrent chronic myelogenous leukemia in marrow transplant patients. Blood 1990;76: 2462-2465.

39 Coulie PG et al.: A new gene coding for a differentiation antigen recognized by autologous cytolytic T lymphocytes on HLA-A2 melanomas. J Exp Med 1994;180:35-42.

40 Galon J et al.: Type, density, and location of immune cells within human colorectal tumors predict clinical outcome. Science 2006;313:1960-1964.

41 Mackensen A et al.: Evidence for in situ amplification of cytotoxic T-lymphocytes with antitumor activity in a human regressive melanoma. Cancer Res 1993;53:3569-3573.
42 Vesely MD et al.: Natural innate and adaptive immunity to cancer. Annu Rev Immunol 2011;29:235271

43 Finn OJ: Cancer vaccines: between the idea and the reality. Nat Rev Immunol 2003;3:630-641.

44 Vansteenkiste $\mathrm{J}$ et al.: Final results of a multicenter, double-blind, randomized, placebo-controlled phase II study to assess the efficacy of MAGEA3 immunotherapeutic as adjuvant therapy in stage IB/II non-small cell lung cancer (NSCLC). American Society of Clinical Oncology (ASCO) Annual Meeting 2007; abstr 7554.

45 Leach DR et al.: Enhancement of antitumor immunity by CTLA-4 blockade. Science 1996;271:17341736.

46 Attia P et al.: Autoimmunity correlates with tumor regression in patients with metastatic melanoma treated with anti-cytotoxic T-lymphocyte antigen-4. J Clin Oncol 2005;23:6043-6053.

47 Hodi FS et al.: Improved survival with ipilimumab in patients with metastatic melanoma. $\mathrm{N}$ Engl J Med 2010;363:711-723.

48 Margolin $\mathrm{K}$ et al.: Ipilimumab in patients with melanoma and brain metastases: an open-label, phase 2 trial. Lancet Oncol 2012;13:459-465.

49 Prieto PA et al.: CTLA-4 blockade with ipilimumab: long-term follow-up of 177 patients with metastatic melanoma. Clin Cancer Res 2012;18:20392047.

50 Ribas A et al.: Antitumor activity in melanoma and anti-self responses in a phase I trial with the anticytotoxic T lymphocyte-associated antigen 4 monoclonal antibody CP-675 206. J Clin Oncol 2005; 23:8968-8977.
1 Beck KE et al.: Enterocolitis in patients with cancer after antibody blockade of cytotoxic T-lymphocyte-associated antigen 4. J Clin Oncol 2006; 24:2283-2289.

52 Zou W, Chen L: Inhibitory B7-family molecules in the tumour microenvironment. Nat Rev Immunol 2008:8:467-477.

53 Topalian SL et al.: Safety, activity, and immune correlates of anti-PD-1 antibody in cancer. N Engl J Med 2012;366:2443-2454.

54 Hanahan D, Weinberg RA: Hallmarks of cancer: the next generation. Cell 2011;144:646-674.

55 Rosenberg SA: Shedding light on immunotherapy for cancer. N Engl J Med 2004;350:1461-1463.

56 June CH: Adoptive T cell therapy for cancer in the clinic. J Clin Invest 2007;117:1466-1476.

57 Robbins PF et al.: Tumor regression in patients with metastatic synovial cell sarcoma and melanoma using genetically engineered lymphocytes reactive with NY-ESO-1. J Clin Oncol 2011;29:917-924.

58 Porter DL et al.: Chimeric antigen receptor-modified T cells in chronic lymphoid leukemia. $\mathrm{N}$ Engl J Med 2011;365:725-733.

59 Hehlmann R, et al.: Tolerability-adapted imatinib $800 \mathrm{mg} / \mathrm{d}$ versus $400 \mathrm{mg} / \mathrm{d}$ versus $400 \mathrm{mg} / \mathrm{d}$ plus interferon- $\alpha$ in newly diagnosed chronic myeloid leukemia. J Clin Oncol. 2011;29:1634-1642. 